\title{
Subsidencia y sus mapas de peligro: Un ejemplo en el área nororiental de la Zona Metropolitana de la Ciudad de México
}

\author{
Enrique Cabral Cano ${ }^{1, *}$, Oscar Díaz Molina ${ }^{1}$, Hugo Delgado Granados ${ }^{2}$ \\ ${ }^{1}$ Departamento de Geomagnetismo y Exploración. Instituto de Geofísica, Universidad Nacional Autónoma de \\ México. México, D.F., 04510, México \\ ${ }^{2}$ Departamento de Vulcanología. Instituto de Geofísica, Universidad Nacional Autónoma de México. México, \\ D.F., 04510. México \\ * ecabral@geofisica.unam.mx
}

\section{Resumen}

El estudio de la magnitud y la variabilidad espacial del proceso de subsidencia mediante el uso de Interferometría de Radar de Apertura Sintética Diferencial en la Ciudad de México nos ha permitido generar un mapa de peligro asociado a la subsidencia. Este trabajo describe la generación de dicho mapa mediante el cálculo del gradiente horizontal de subsidencia y presenta un ejemplo de esta metodología en el área nororiental de la Zona Metropolitana de la Ciudad de México.

Palabras clave: DInSAR, subsidencia, peligro, México.

\begin{abstract}
Analysis of subsidence magnitude and its spatial variability through Differential Interferometric Synthetic Aperture Radar techniques in Mexico City has allowed us to generate a subsidence-related faulting hazard cartographic product. This study describes the generation of hazard maps through the derivation of the horizontal subsidence gradient and presents an example of this methodology on the northeastern area of the Mexico City Metropolitan Zone.
\end{abstract}

Keywords: DInSAR, subsidence, hazard, Mexico

\section{Introducción}

La técnica de InSAR ha demostrado ser una excelente herramienta para el análisis de la magnitud y la variabilidad espacial del proceso de hundimiento en nuestro país (Farina et al., 2007; Ávila-Olivera, 2008; Cabral-Cano et al., 2008, López-Quiroz, 2009). Las principales ventajas de este método radican en que a) brinda una visión sinóptica del proceso de hundimiento y b) permite generar productos que poseen una alta resolución espacial que difícilmente puede ser obtenida por métodos de nivelación convencional.

Las tasas de extracción de agua subterránea en la cuenca de México (Figura 1) han provocado un abatimiento de su nivel estático que va de 0.1 a $1.5 \mathrm{~m} /$ año (JACMCWS, 1995). Esto ocasiona la reducción de la presión de fluido en los poros, la compactación de arcillas y finalmente subsidencia del suelo. Los efectos de este proceso, aunque han sido reconocidos por más de un siglo (p. ej. Gayol, 1925; Carrillo, 1948; CHCVM, 1953; CAVM, 1975; Figueroa 


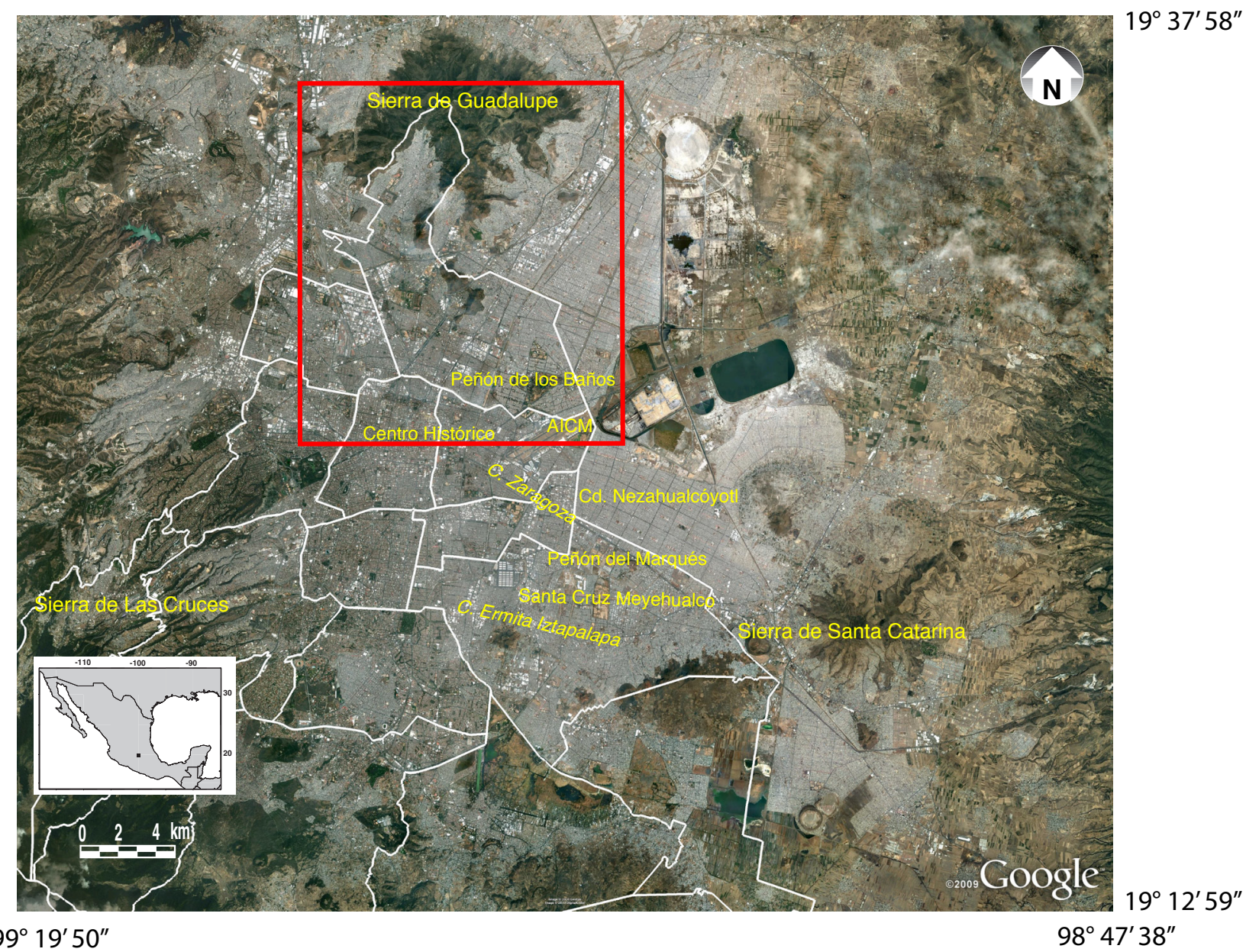

Figura 1. Mapa de localización del área de estudio (recuadro rojo) dentro de la Zona Metropolitana de la Ciudad de México. Imagen tomada de Google Earth. Aeropuerto Internacional de la Ciudad de México (AICM).

Vega, 1984; Ortega et al., 1993), se han incrementado en los últimos 50 años debido a la rápida urbanización de la cuenca de México a partir de la década de 1970.

Los estudios de subsidencia en la Zona Metropolitana de la Ciudad de México utilizando Interferometría de Radar de Apertura Sintética Diferencial (DInSAR) y GPS (Cabral-Cano et al., 2007, 2008), indican que la subsidencia en la Zona Metropolitana de la Ciudad de México desde el inicio de la existencia de datos SAR, en 1996, presenta tasas que rebasan los $-370 \mathrm{~mm} /$ año en su zona oriente $(i$. $e$. Ciudad Nezahualcóyotl), mientras que el área del centro histórico muestra tasas de hundimiento que varían de -92 a $-115 \mathrm{~mm} /$ año (Figura 2). Para poner en contexto este hecho es suficiente mencionar que dichas tasas de subsidencia son muy cercanas a los $-400 \mathrm{~mm} / \mathrm{año} \mathrm{detectados} \mathrm{en} \mathrm{la} \mathrm{dé-}$ cada de 1950, cuando se tomaron las primeras acciones de mitigación que incluyeron una veda de extracción de agua en pozos del centro de la ciudad y el eventual desarrollo de la red de pozos de extracción en la periferia.

La cartografía derivada de este tipo de análisis puede proveer elementos de decisión que resultan de vital im- portancia para la cuantificación del potencial daño a la infraestructura urbana de servicios, estructuras civiles y de comunicación debido al hundimiento y su fallamiento asociado. Ejemplos del uso de InSAR (p. ej. Osmanoglu et al., 2010; Cabral-Cano et al., 2010a y b) y de geofísica de exploración somera (Giordano et al., 2010) muestran el vínculo subsidencia-fallamiento en zonas que presentan altas tasas anuales de subsidencia. Debido a la falta de un análisis espacial detallado previo de este fenómeno, los resultados cartográficos derivados de este estudio proporcionan a las administraciones de gobierno una valiosa herramienta para desarrollar de manera integral nuevos planes de protección civil, de desarrollo y uso de suelo y de mantenimiento de infraestructura urbana.

Este trabajo muestra los resultados de un ejemplo de la utilización de InSAR para generar mapas de peligro relativo en el área nororiental de la Zona Metropolitana de la Ciudad de México que permitan una mejor planeación, tanto de actividades de mitigación como de mejoramiento del uso de suelo en áreas de alta vulnerabilidad. 
a)

1996

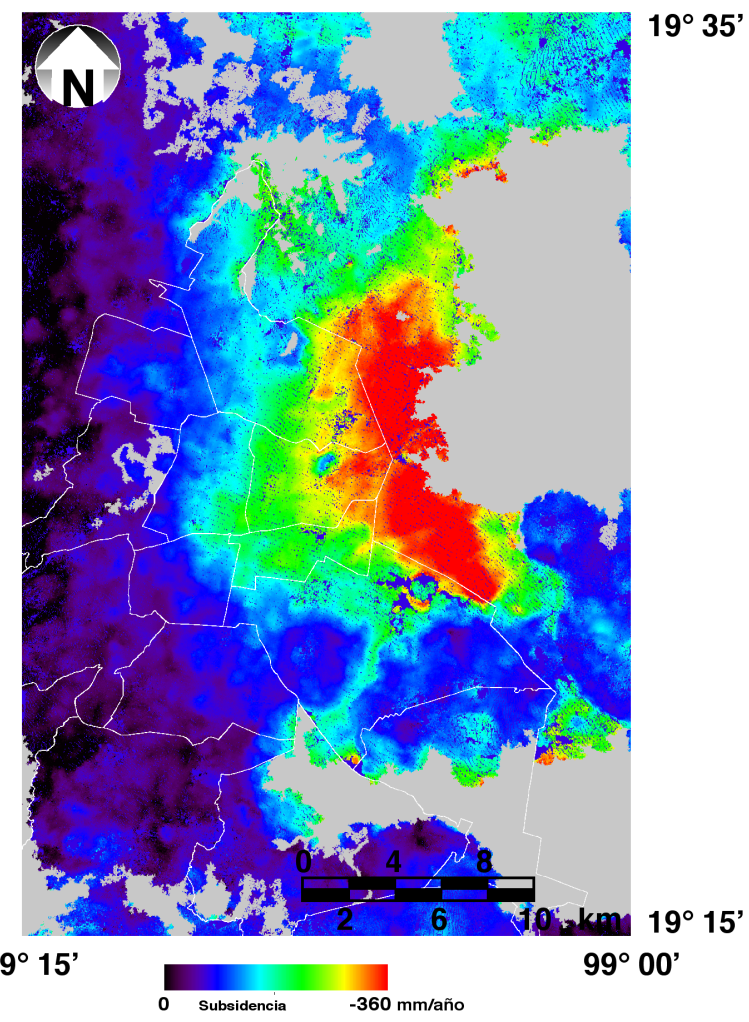

c)

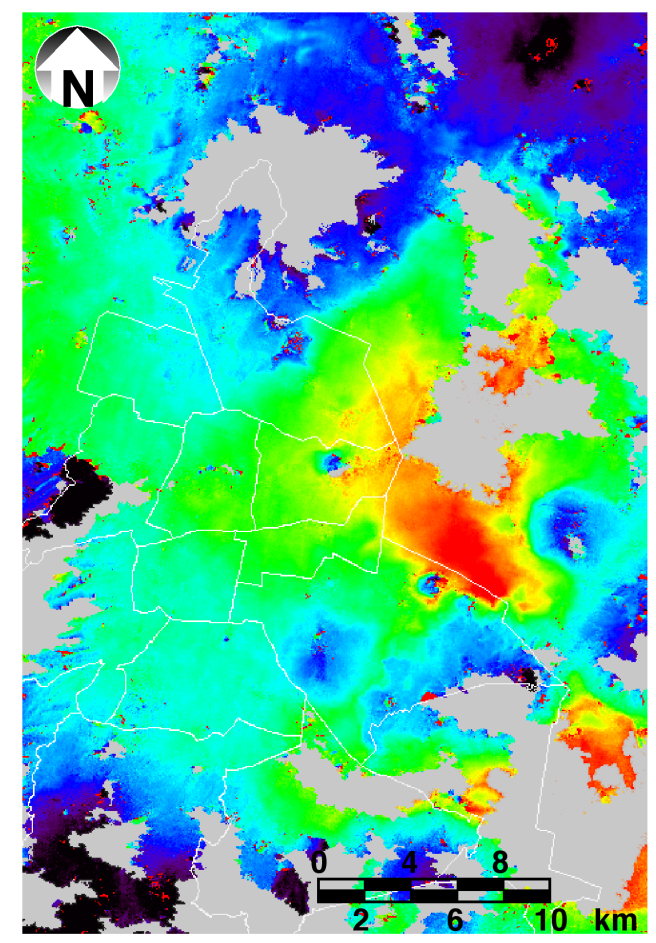

b) 2000

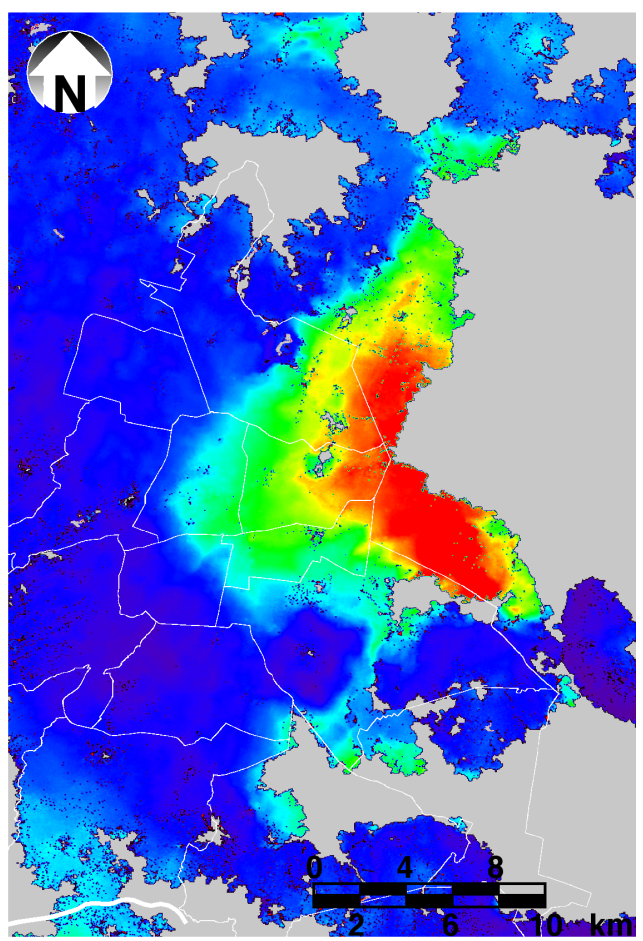

d)

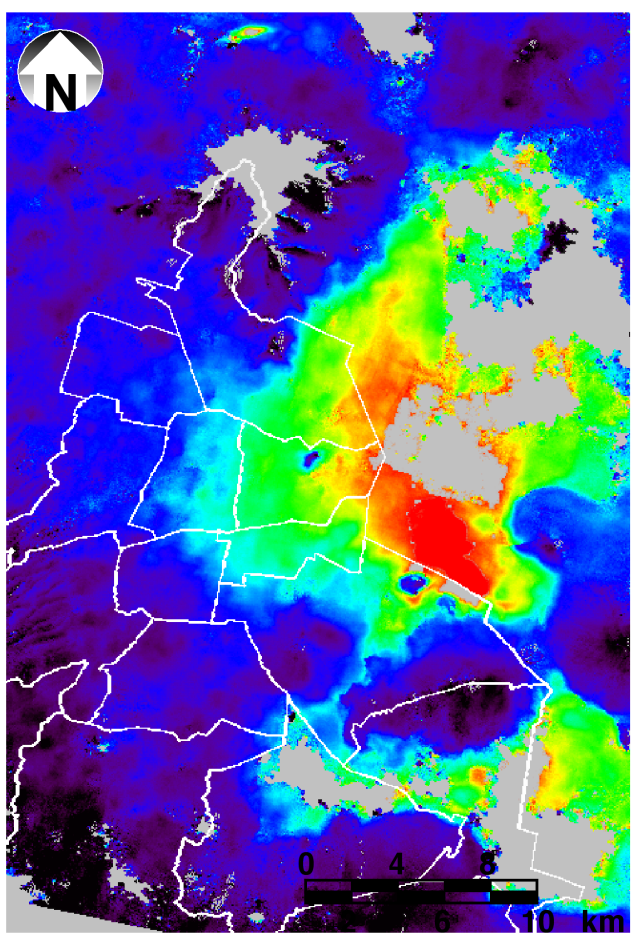




\section{El gradiente horizontal de subsidencia en la Zona Metropolitana de la Ciudad de México}

Los avances en la cuantificación de la magnitud de subsidencia en varias ciudades de México (e.g. Cabral-Cano et al., 2010a y b) muestran que la magnitud de subsidencia no es necesariamente el factor más importante al evaluar la vulnerabilidad de la infraestructura urbana al desarrollo de fallamiento superficial, sino que la principal condicionante de peligro por fracturamiento se manifiesta en aquellas zonas donde se observa un alto gradiente horizontal de subsidencia.

La resolución espacial inherente al procesamiento InSAR de escenas ERS-1/2 o ENVISAT-ASAR, permite obtener el gradiente horizontal en áreas unitarias del orden de las decenas de metros a partir de las observaciones de desplazamiento a lo largo de la línea de vista del instrumento. Esto se logra mediante el cálculo de la diferencia de valores entre cada uno de los píxeles de la imagen de magnitud de subsidencia, dividido entre la distancia horizontal entre píxeles. Este cálculo se realiza para cada uno de los píxeles de las imágenes de magnitud de subsidencia y se determina el valor máximo encontrado. La tasa de cambio resultante, definida como la variación en la magnitud de hundimiento por unidad de longitud horizontal (Cabral-Cano et al., 2007), se puede representar en forma de imágenes georeferenciadas que permiten realizar clasificaciones del nivel potencial de peligro con alta resolución espacial.

Las ventajas de los mapas generados a partir del análisis InSAR, en especial los de gradiente horizontal, radican en que es posible detectar movimientos diferenciales que generan esfuerzos de cizalla en la superficie del suelo, en estructuras civiles o en ductos y tuberías subterráneas. Por otra parte, los mapas de gradiente de subsidencia no son mapas de ocurrencia potencial de un fenómeno, sino que se generan directamente de las mediciones de un proceso geológico, que es una condicionante de peligro. Esto les da la posibilidad de tener un carácter predictivo.

Aunque la cuantificación de la magnitud de subsidencia mediante InSAR en la Zona Metropolitana de la Ciudad de México observada en el periodo 1996-2005 puede presentar ligeras diferencias en su distribución espacial, todos los mapas para este periodo muestran patrones característicos que delimitan claramente cuatro principales zonas que presentan un alto gradiente horizontal (Figura 2):

1) Ladera sur de la Sierra de Guadalupe, al norte de la Ciudad de México.

2) Peñón de los Baños, inmediatamente al norte del Aeropuerto Internacional de la Ciudad de México.

3) El corredor de la calzada Zaragoza, que tiene una orientación paralela a esta vialidad incluyendo la zona del Peñón del Marqués, en el sector oriental de la zona metropolitana.

4) El corredor con dirección NE-SW localizado en las inmediaciones de la colonia Santa Cruz Meyehualco, al norte de la calzada Ermita Iztapalapa.

\section{Subsidencia en el área nororiental de la Zona} Metropolitana de la Ciudad de México

Para el caso del análisis de subsidencia en el área nororiental de la Zona Metropolitana de la Ciudad de México ( $i$. $e$. al sur de la Sierra de Guadalupe y al norte del Peñón de los Baños) se generaron 4 mapas de desplazamiento mediante técnicas de DInSAR (Figura 3) correspondientes a los periodos 15/04/2005-24/06/2005, 21/09/2005-26/10/2005, 20/01/2006-24/02/2006 y 05/05/2006-09/06/2006, que representan las soluciones que mantienen mejor coherencia en el proceso InSAR. El procesamiento realizado incluyó la creación de imágenes complejas de vista sencilla (Single Look Complex: SLC), el registro de ambas imágenes SLC, el cálculo de interferogramas utilizando la técnica de doble paso+DEM, el filtrado, el desdoblamiento de fase y la generación de mapas de desplazamiento. A partir de estos mapas se generaron productos complementarios, como mapas de gradiente horizontal de subsidencia (CabralCano et al., 2007; Figura 4) y clasificaciones relativas de peligro con base en el gradiente de subsidencia (Figura 5).

El análisis regional de hundimiento por InSAR para el periodo 2005-2006 permitió detectar varias zonas que presentan altas tasas de subsidencia en el área de estudio (Figura 3). Los mayores valores se observan en la zonas del Aeropuerto Internacional y Bosques de Aragón, con valores mayores a los $-350 \mathrm{~mm} /$ año, mientras que las zonas de valores de hundimiento intermedios de entre - 100 y 200 $\mathrm{mm} /$ año se localizan al este del Eje 1 Oriente. Sin embargo, las áreas con valores altos de gradiente horizontal en el área de estudio (Figura 4), y que por lo tanto se consideran más vulnerables a experimentar daños, se localizan en:

a) La vertiente sur de la Sierra de Guadalupe, en especial en las laderas noroeste, oeste y sur del cerro Chiquihuite y la ladera este del Puerto el Panal.

b) Las laderas sur y este del parque Tepeyac, al norte de la Av. Martín Carrera y al oeste de la Av. Eduardo Molina.

c) El flanco norte del Peñón de los Baños en el área de la Unidad San Juan de Aragón.

d) La zona al sur del deportivo Galeana y que incluye al bosque de San Juan de Aragón.

e) El corredor en dirección noreste que se encuentra al noreste del Parque Nacional Tepeyac y delimitado entre el Gran Canal del Desagüe y la Vía Morelos.

Dado que existe una clara correlación entre daños superficiales en redes hidráulicas, estructuras civiles y demás infraestructura urbana y el gradiente horizontal de subsidencia (Cabral-Cano et al., 2010a), es posible determinar zonas de peligro a partir de los mapas de gradiente horizontal de subsidencia. Aunque se pueden definir los umbrales para las diferentes clases de peligro con base en la capacidad de las estructuras civiles para resistir esfuerzos de cizalla, la zona de estudio es heterogénea, donde las construcciones siguiendo códigos estrictos de construcción coexisten con casas habitación donde los procesos de autoconstrucción y la calidad de la construcción son muy 
a) $2005 / 04 / 15-2005 / 06 / 24$

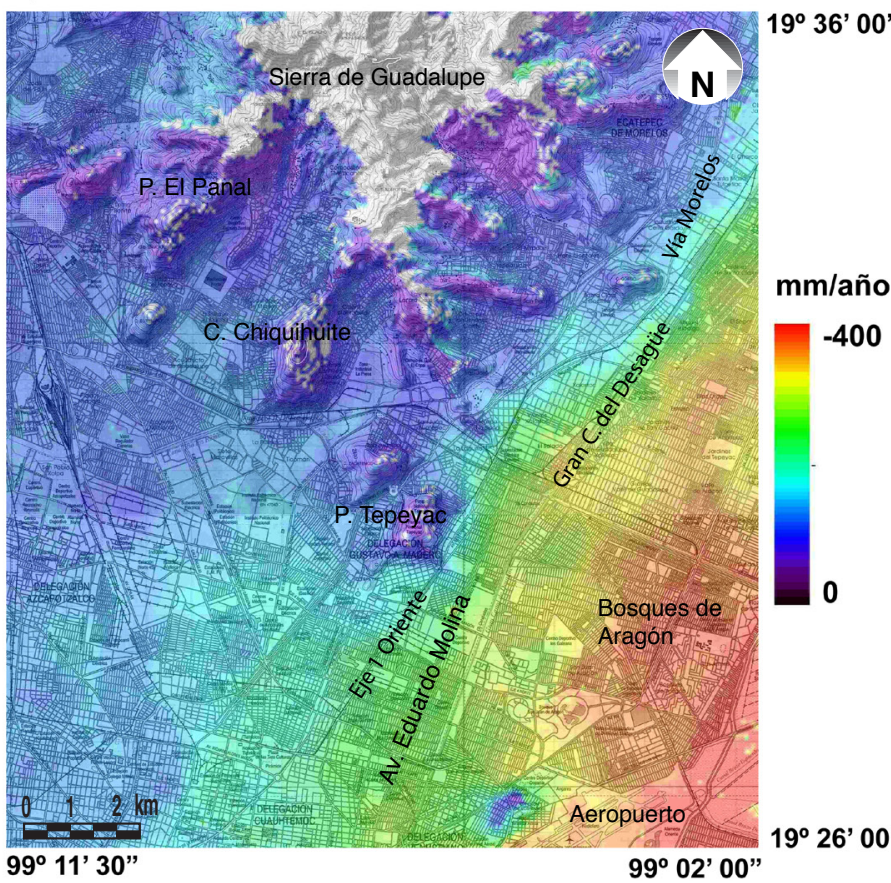

b) $2005 / 09 / 21-2005 / 10 / 26$

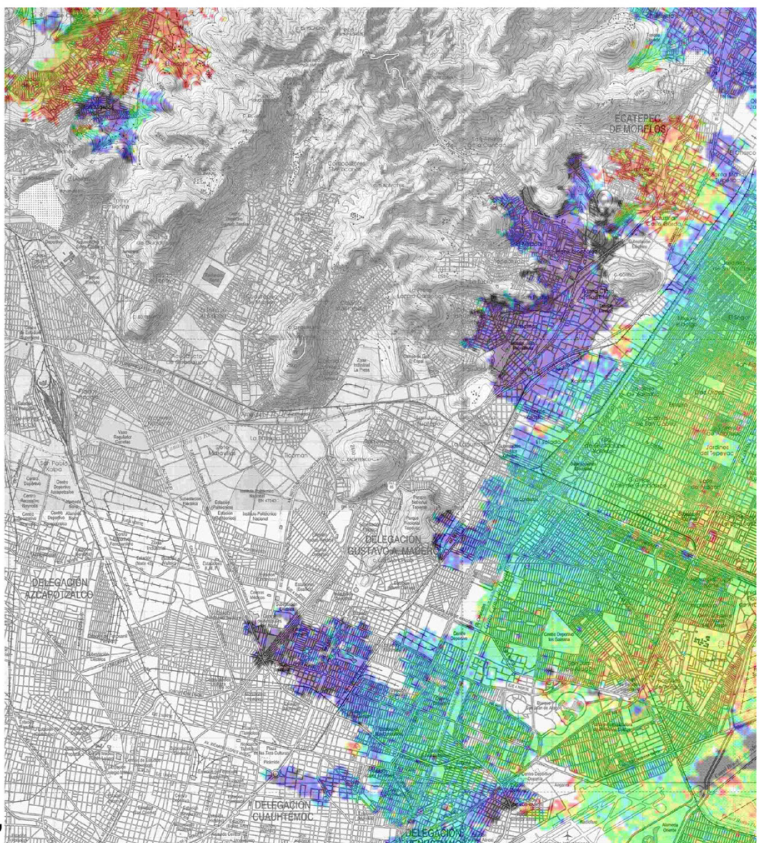

\section{c) $2006 / 01 / 20-2006 / 02 / 24$}

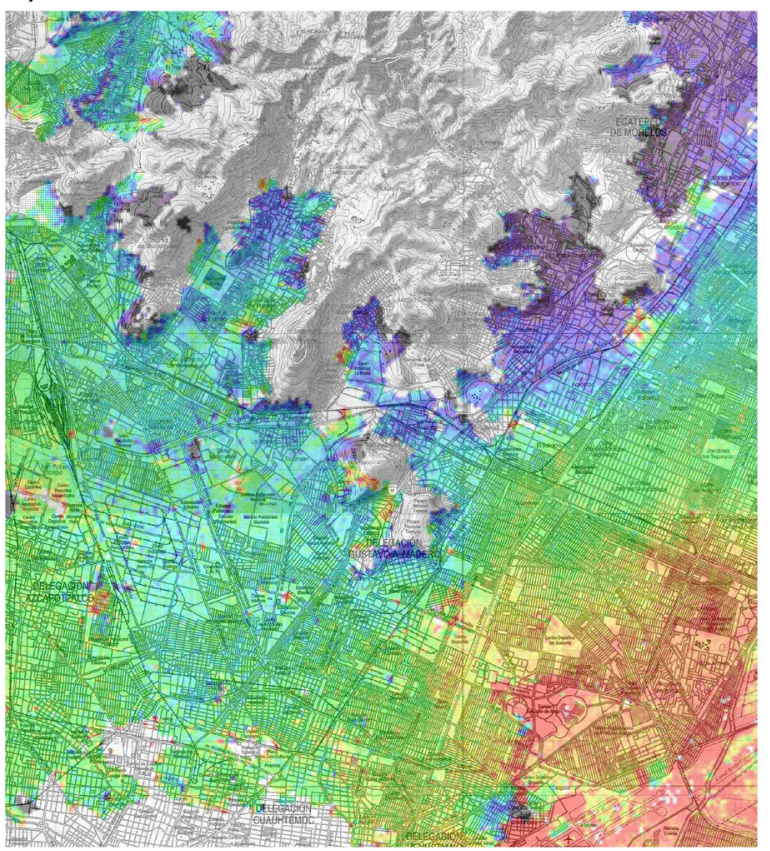

d) $2006 / 05 / 05-2006 / 06 / 09$

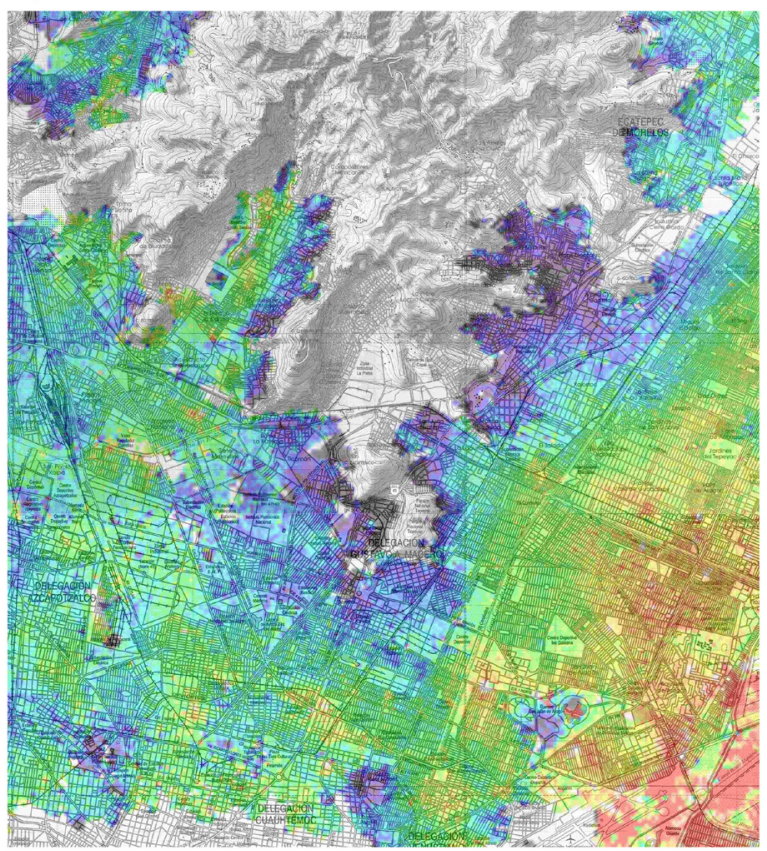

Figura 3. Mapas de tasas de desplazamiento vertical generados por técnicas de interferometría diferencial para diferentes periodos comprendidos enre abril de 2005 y junio de 2006. Los valores de subsidencia se encuentran normalizados y se expresan en mm/año, para facilitar su intercomparación.

variables. En vista de estas circunstancias, y del rango tan amplio de la magnitud de subsidencia, se ha optado por una simple división lineal para definir los umbrales de las áreas de peligro bajo, medio y alto (Figura 5). En este mapa de peligro se muestran de manera gráfica, pero con una gran resolución espacial, las áreas consideradas críticas, donde los planes de desarrollo y de uso del suelo deberían incorporar estos nuevos criterios y evitar en la medida de lo posible la construcción de edificios públicos como escuelas y similares. 
a) $2005 / 04 / 15-2005 / 06 / 24$

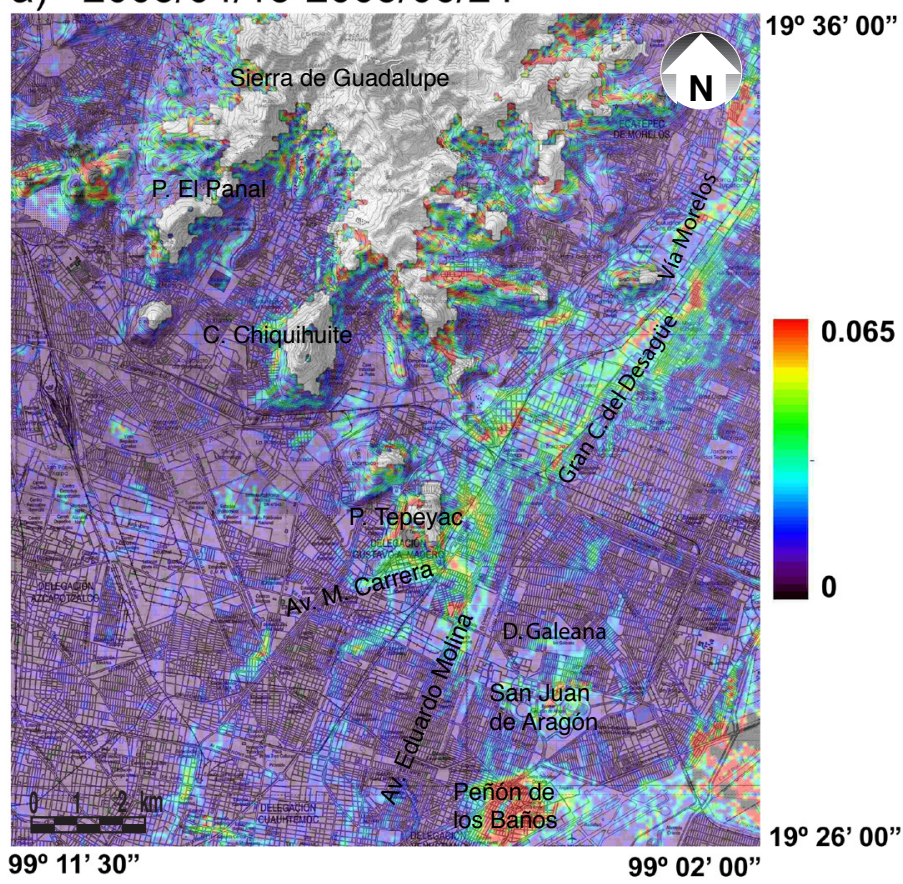

b) $2005 / 09 / 21-2005 / 10 / 26$

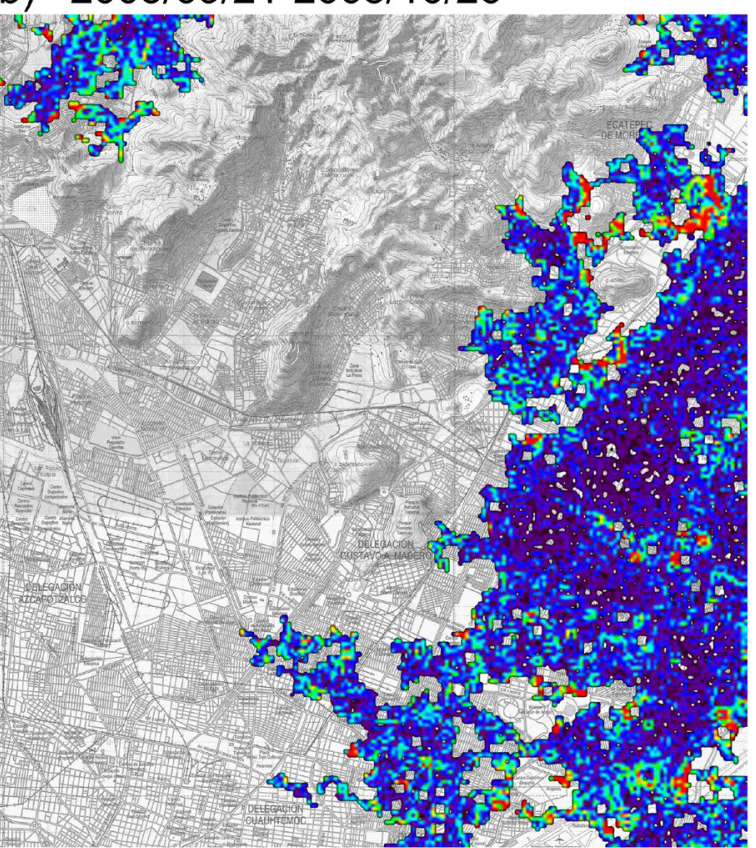

c) $2006 / 01 / 20-2006 / 02 / 24$

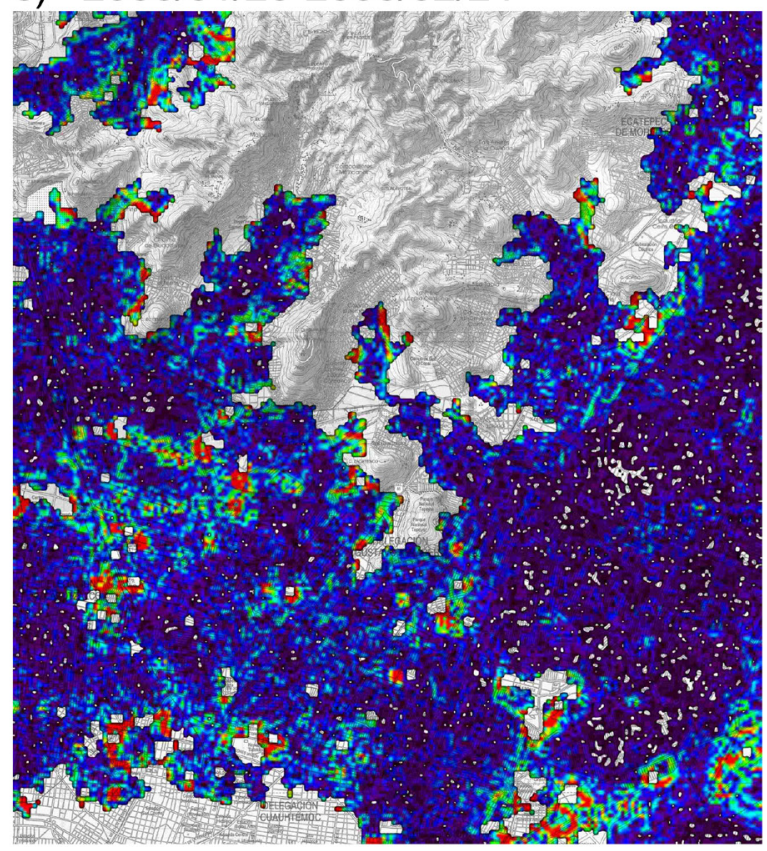

d) $2006 / 05 / 05-2006 / 06 / 09$

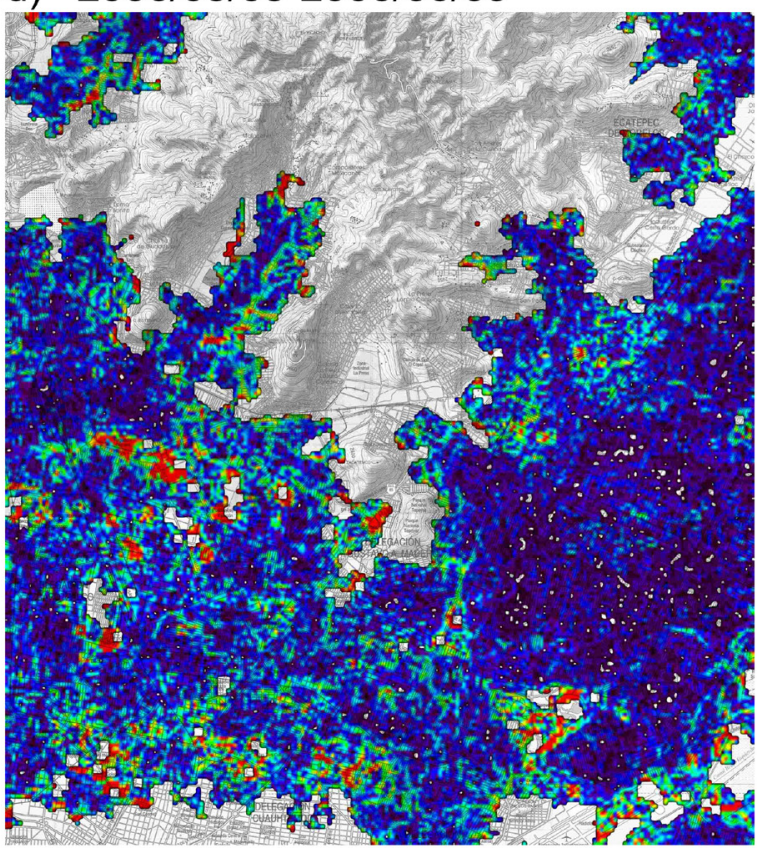

Figura 4. Mapas de gradiente horizontal de subsidencia para periodos comprendidos entre abril de 2005 y junio de 2006.

\section{Conclusiones}

Los resultados del análisis de DInSAR aquí presentados proveen elementos de decisión de vital importancia para la cuantificación del potencial de daño a la infraestructura urbana de servicios y estructuras civiles, debido al proceso de hundimiento que experimenta un área significativa de la Zona Metropolitana de la Ciudad de
México. Los resultados que aquí se muestran constituyen una herramienta de gran utilidad, al permitir incorporar el proceso de subsidencia y su fallamiento asociado como un elemento de consideración más en los planes de protección civil, de mantenimiento de infraestructura urbana y en los escenarios de desarrollo y uso de suelo. 


\section{$2005 / 04 / 15-2005 / 06 / 24$}

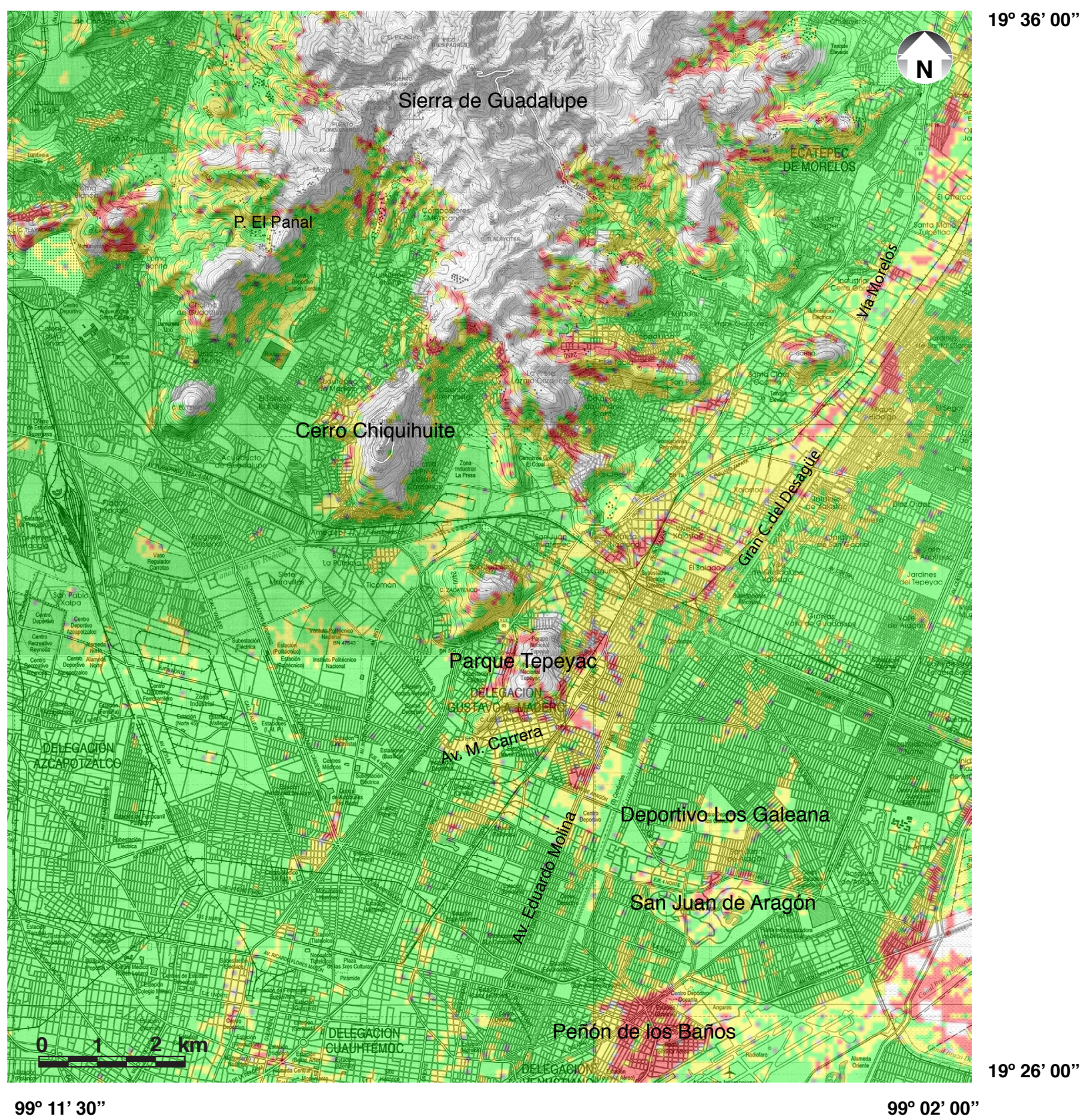

$99^{\circ} 11^{\prime} 30^{\prime \prime}$

$99^{\circ} 02^{\prime} 00^{\prime \prime}$

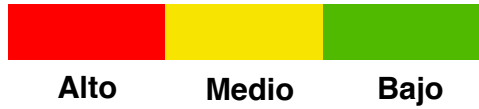

Figura 5. Clasificación relativa de peligro por fallamiento asociado a subsidencia generado a partir del gradiente horizontal de subsidencia en la zona nororiental de la Zona Metropolitana de la Ciudad de México.

\section{Agradecimientos}

Este trabajo fue apoyado por los proyectos UNAM-PAPIIT IN-121515, IN-114907 e IN117909 y Conacyt 61212 y 82868. Los datos Envisat fueron obtenidos a través de la Agencia Espacial Europea (ESA) por medio del proyecto CAT-1 1409.

\section{Referencias}

Ávila Olivera, J.A., 2008, Evolución de los procesos de subsidenciacreep-falla, casos: Morelia, Mich. y Celaya, Gto.: México, D. F., Universidad Nacional Autónoma de México, tesis doctoral, 249 p.

Cabral-Cano, E., Dixon, T., Diaz-Molina, O., 2007, InSAR derived horizontal subsidence gradient as a tool for hazard assessment in urban areas (abstract), en Eos Transactions, American Geophysical Union 
88 Joint Assembly Supplement, G43B-13.

Cabral-Cano, E., Dixon, T.H., Miralles-Wilhelm, F., Díaz-Molina, O., Sánchez-Zamora, O., Carande, R.E., 2008, Space geodetic imaging of rapid ground subsidence in Mexico City: Bulletin of the Geological Society of America, 120, 1556-1566.

Cabral-Cano, E., Osmanoglu, B., Dixon, T., Wdowinski, S., DeMets, C., Cigna, F., Díaz-Molina, O., 2010a, Subsidence and faulting hazard maps using PSI and permanent GPS networks in central Mexico, en Eighth International Symposium on Land Subsidence: Querétaro, México, International Association of Hydrological Sciences Publication, 339, 255-259.

Cabral-Cano, E., Arciniega-Ceballos, A., Díaz-Molina, O., Cigna, F., Ávila-Olivera, A., Osmanoglu, B., Dixon, T.H., Demets, C., Garduño-Monroy, V.H., Vergara-Huerta, F., Hernández-Quintero, J.E., 2010b, Is there a tectonic component to the subsidence process in Morelia, Mexico?, en Eighth International Symposium on Land Subsidence: Querétaro, México, International Association of Hydrological Sciences Publication, 339, 164-169.

Carrillo, N., 1948, Influence of artesian wells on the sinking of México City, en Proceedings of the Second International Conference on Soils Mechanics: Rotterdam, Holanda, International Society for Soil Mechanics and Geotechnical Engineering, VII.

Comisión de Aguas del Valle de México (CAVM), 1975, Boletín de Mecánica de Suelos No.7: México, D.F. Comisión de Aguas del Valle de México-SARH, $289 \mathrm{p}$

Comisión Hidrológica de la Cuenca del Valle de México (CHCVM), 1953, Boletín de Mecánica de Suelos No. 1: México, D.F., Comisión de Aguas del Valle de México-SRH.

Farina, P., Avila-Olivera, J.A., Garduño-Monroy, V.H., 2007, Structurally-controlled urban subsidence along the Mexican Volcanic Belt (MVB) monitored by InSAR, en Proceedings of Envisat Symposium 2007: Montreux, Suiza, European Space Agency, 6 p.

Figueroa-Vega, F., 1984, Case history No. 9.8, Mexico, D.F., Mexico, en Poland, J.F. (ed.), Guidebook to studies of land subsidence due to ground-water withdrawal: Paris, Francia, United Nations Educational Scientific and Cultural Organization, 217-232.

Gayol, R., 1925, Estudio de las perturbaciones que en el fondo de la Ciudad de México ha producido el drenaje de las aguas del subsuelo, por las obras del desagüe y rectificación de los errores a que ha dado lugar una incorrecta interpretación de los efectos producidos: Revista Mexicana de Ingeniería y Arquitectura, III, 96-132.

Giordano, N., Díaz-Molina, O., Cabral-Cano, E., Garduño Monroy, V.H., Hernández-Madrigal, V.M., Camargo-Valencia, A., 2010, The application of ground penetrating radar in the study of subsidencecreep induced faults in Celaya (resumen), en Eighth International Symposium on Land Subsidence: Querétaro, México, International Association of Hydrological Sciences Publication, 8.

Joint Academies Committee on the Mexico City Water Supply (JACMCWS), 1995, Mexico City's water supply, improving the outlook for sustainability: Washington, D.C., National Academy Press, $256 \mathrm{p}$.

López-Quiroz, P., Doin, M.P., Tupin, F., Briole, P., \& Nicolas, J.M., 2009, Time series analysis of Mexico 582 City subsidence constrained by radar Interferometry. Journal of Applied Geophysics, 69, 1-15.

Ortega-Guerrero, A., Cherry, J.A., Rudolph, D.L., 1993, Large-scale aquitard consolidation near Mexico City: Ground Water, 31, 708-718.

Osmanoglu, B., Dixon, T.H., Wdowinski, S., Cabral-Cano, E., Jiang, Y., 2011, Mexico City subsidence observed with persistent scatterer InSAR: International Journal of Applied Earth Observation and Geoinformation, 13, 1-12.

Manuscrito recibido: Octubre 04, 2009

Manuscrito corregido recibido: Febrero 12, 2010

Manuscrito aceptado: Febrero 22, 2010 